\title{
Inducible expression of filaggrin increases keratinocyte susceptibility to apoptotic cell death
}

\author{
MK Kuechle ${ }^{\star 1,2}$, RB Presland ${ }^{1,2}$, SP Lewis ${ }^{1}$, P Fleckman $^{2}$ and \\ BA Dale ${ }^{1,2,3,4}$ \\ ${ }^{1}$ Department of Oral Biology, University of Washington, Seattle, Washington, \\ WA 98195, USA \\ 2 Department of Medicine (Dermatology), University of Washington, Seattle, \\ Washington, WA 98195, USA \\ ${ }^{3}$ Department of Periodontics, University of Washington, Seattle, Washington, \\ WA 98195, USA \\ ${ }^{4}$ Department of Biochemistry, University of Washington, Seattle, Washington, \\ WA 98195, USA \\ * Corresponding author: MK Kuechle, Departments of Oral Biology/Medicine \\ (Dermatology), Box 357132, University of Washington, Seattle, Washington, \\ WA 98185-7132, USA. Tel: (206) 543-1595; Fax: (206) 685-3162; \\ E-mail:kuechles@u.washington.edu
}

Received 11.8.99; revised 10.1.00; accepted 6.3.00

Edited by JC Reed

\begin{abstract}
Filaggrin is an intermediate filament associated protein that aids the packing of keratin filaments during terminal differentiation of keratinocytes. Premature aggregation of keratin filaments is prevented by filaggrin expression as the inactive precursor, profilaggrin, which is localized in keratohyalin granules in vivo. We have previously shown that filaggrin constructs, when transiently transfected into epithelial cells, lead to a collapsed keratin cytoskeletal network and dysmorphic nuclei with features of apoptosis. The apparent transfection rate is low with filaggrin constructs, supporting their disruptive role but hindering further study. To bypass this problem, we generated stable keratinocyte cell lines that express mature human filaggrin using a tetracyclineinducible promoter system. We found that cell lines expressing filaggrin, but not control cell lines, exhibited increased sensitivity to multiple apoptotic stimuli as measured by morphologic and biochemical criteria. None of the cell lines showed an increase in endogenous expression of filaggrin in response to the same stimuli. Filaggrin expression alone was insufficient to induce apoptosis in these keratinocyte cell lines. We conclude that filaggrin, due to its keratin binding ability, primes cells for apoptosis. Because filaggrin is expressed at a level of the epidermis where keratinocytes are in transition between the nucleated granular and the anucleate cornified layers, we hypothesize that filaggrin aids in the terminal differentiation process by facilitating apoptotic machinery. Cell Death and Differentiation (2000) 7, 566-573.
\end{abstract}

Keywords: filaggrin; apoptosis; keratinocyte; differentiation

Abbreviations: DAPI, 4', 6-diamidino-2-phenylindole dihydrochloride; DMEM, Dulbecco's modified Eagle medium; EDTA, ethylene diamine tetra-acetic acid; GFP, green fluorescent protein; PBS, phosphate buffered saline; REK, rat epidermal keratinocyte; TBS, tris buffered saline; TUNEL, terminal deoxytransferase-mediated dUTP-biotin nick end labeling

\section{Introduction}

The stratum corneum is the thin $(12-15 \mu \mathrm{m})$ tough, outermost layer of the epidermis composed of overlapping, flattened, corneocytes and lipid-rich, intercellular lamellae. ${ }^{1}$ The stratum corneum functions as a barrier both to keep environmental insults out and to prevent water loss. Many morphologic and biochemical changes occur during the formation of the stratum corneum. Loricrin, involucrin, the small proline-rich proteins (SPR's), and other substrates are crosslinked by transglutaminase, while the intermediate filament network is reorganized into compact macrofibrils oriented parallel to the body surface. ${ }^{2}$

Filaggrin is a highly charged, cationic protein that aids aggregation and subsequent disulfide bonding of keratin filaments. ${ }^{3}$ It is derived from profilaggrin, a large ( $>400 \mathrm{kD}$ ), phosphorylated precursor expressed as keratohyalin granules in the granular layer of the epidermis. During the transition from the granular layer to the stratum corneum, profilaggrin is converted to filaggrin by site-specific proteolysis and dephosphorylation. ${ }^{4-7}$ In addition to profilaggrin processing to filaggrin, the transition from a granular cell to a corneocyte is characterized by the degradation of the nucleus and other organelles, assembly of a cornified envelope, and reorganization of the keratin intermediate filament network into a twodimensional sheet.

The morphologic changes of the keratinocyte at the transition have suggested that terminal differentiation is a specialized form of apoptosis. ${ }^{8,9}$ A small percentage of transition cells exhibit fragmented DNA in situ ${ }^{10,11}$ and evidence of endonuclease activity. ${ }^{12}$ However, differences exist between apoptosis and terminal differentiation, and the two processes have been shown to be subject to different and distinct stimuli. ${ }^{13}$ Caspases, a family of cysteinyl aspartate proteases, are considered the effector enzymes for apoptosis. ${ }^{14,15}$ Many caspases are expressed in the epidermis, ${ }^{16}$ and caspase-3 has been shown to be expressed in the active form at the granular layer. ${ }^{17}$ It is not known if other caspases are activated at the transition to the stratum corneum.

We have previously shown that transient expression of filaggrin in epithelial cells leads to a phenotype of apoptosis with cell contraction, nuclear membrane breakdown, and nuclear condensation. ${ }^{18}$ Transient expression of profilaggrin-like constructs, the precursor of filaggrin, leads to expression of a granular protein that does not have the same toxic effects as mature filaggrin on the cell. Because recovery of transfectants is very low with 
the filaggrin construct $(<2 \%)$ we were unable to obtain biochemical support for apoptosis. To circumvent the low transfection rate, the effects of filaggrin expression were examined in a tetracycline-inducible rat keratinocyte cell line. ${ }^{19}$ Filaggrin expression in this system does not result in measurable levels of apoptosis, but instead leads to cell cycle arrest.

In the present study, we test the hypothesis that filaggrin expressing lines are more susceptible to death-inducing stimuli. The biologic relevance of this question relies on the fact that filaggrin is expressed at a point in epidermal differentiation where the keratinocytes lose their nuclei and other organelles, collapse upon themselves, and cease synthetic activity, i.e. all aspects of apoptosis. We show in this study that filaggrin expression facilitates induction of apoptosis in vivo. These results suggest that filaggrin may function in vivo to facilitate aspects of terminal differentiation that utilize apoptotic mechanisms.

\section{filaggrin}
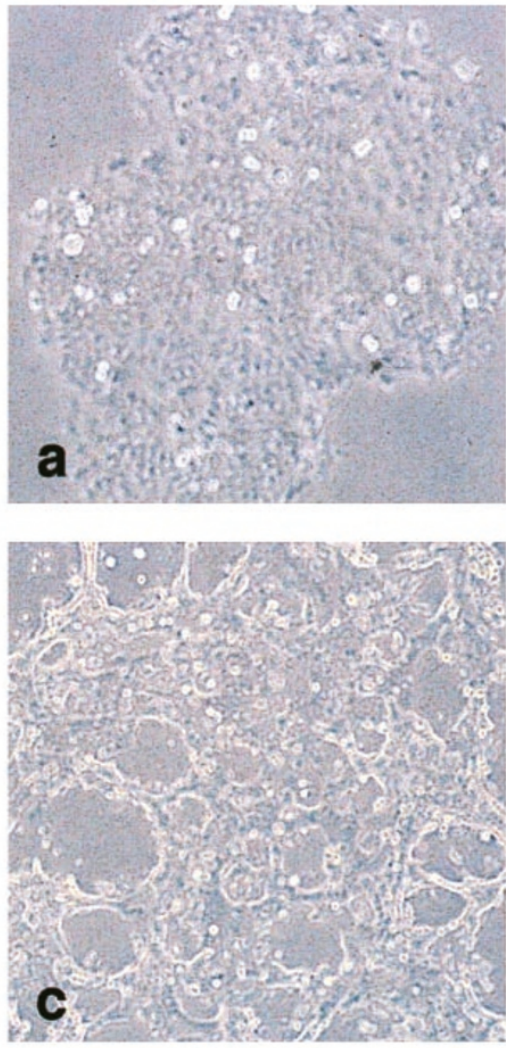

\section{$25 \mathrm{mj}$ UVB}

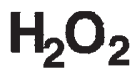

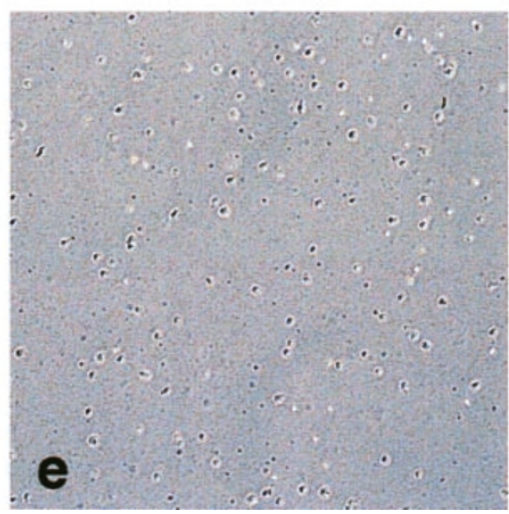

\section{B-galactosidase}
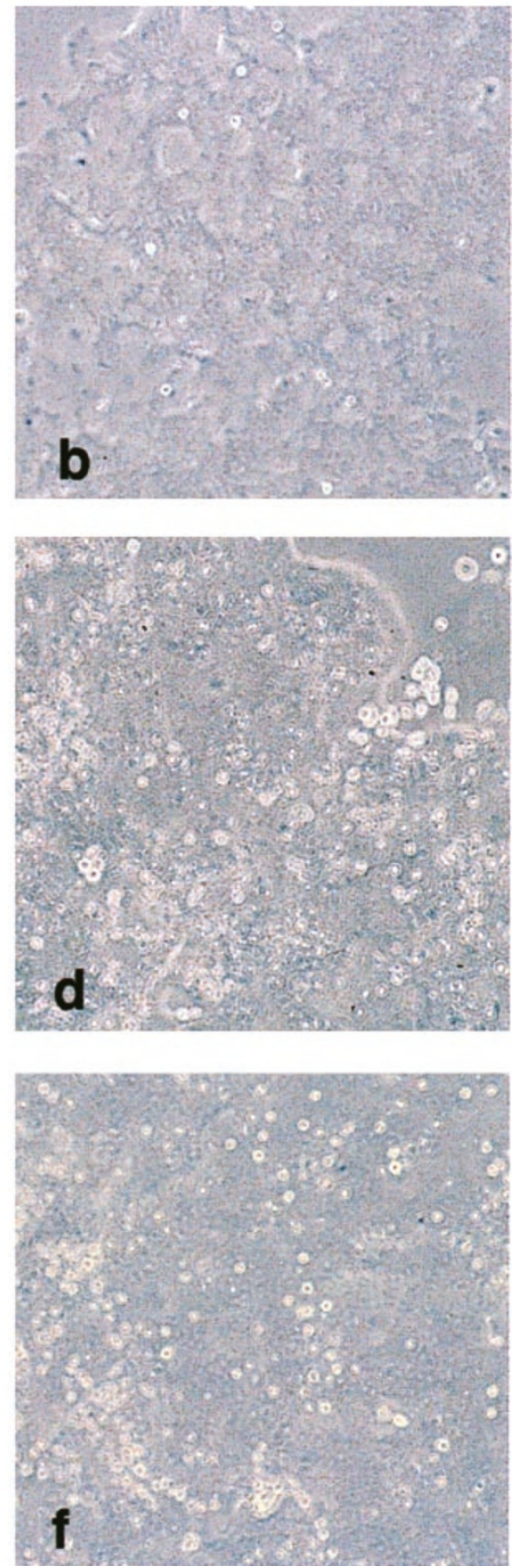

Figure 1 Filaggrin expressing cell lines (2F5) round up and lose cell contacts with lower doses of UVB and $\mathrm{H}_{2} \mathrm{O}_{2}$ than $\beta$-gal expressing cell lines (3B10). Phase contrast photomicrographs of stable rat keratinocytes that express either filaggrin $(\mathbf{a}, \mathbf{c}, \mathbf{e})$ or $\beta$-galactosidase $(\mathbf{b}, \mathbf{d}, \mathbf{f})$ with no apoptotic stimulus $(\mathbf{a}, \mathbf{b}), 4 \mathrm{~h}$ after exposure to $25 \mathrm{mj}$ of UVB (c,d), or exposure to $100 \mu \mathrm{M}$ hydrogen peroxide for $4 \mathrm{~h}(\mathbf{e}, \mathbf{f})$. No appreciable difference is seen between the filaggrin expressing and $\beta$ galactosidase expressing cells in control cultures. However, a marked difference is seen between the two cell lines after exposure to UVB and $\mathrm{H}_{2} \mathrm{O}_{2}$, as the filaggrin expressing cells are rounded up and losing cell contacts 


\section{Results \\ Inducible expression of filaggrin does not cause apoptosis in rat keratinocytes}

We had previously shown that transient expression of filaggrin in epithelial cells leads to a phenotype suggestive of apoptosis; ${ }^{18}$ however, because of the low frequency of filaggrin positive cells, it was not possible to confirm this finding. The low transfection rate is also seen with filaggrinGFP constructs ( $<2 \%$ ), compared to $15-20 \%$ for GFP and $\beta$ galactosidase controls. ${ }^{20}$ The disparity in transfection rate between filaggrin and other constructs suggested to us that filaggrin expression was toxic to the cell. To overcome this problem and to enable us to further study the effects of filaggrin expression in epithelial cells, we developed an inducible system based on the tetracycline promoter. ${ }^{19}$ Using this method, cells were induced to express filaggrin by addition of doxycycline for periods of $1-7$ days. Apoptosis was evaluated by morphologic and biochemical criteria. Filaggrin expressing cells did not differ from $\beta$-galactosidase expressing cells in any of the apoptotic assays at any time point examined (Figures 1a,b, 2b,d, 3a,d, 4).

\section{Filaggrin, but not $\beta$-galactosidase, expression makes rat keratinocytes more susceptible to apoptosis}

One possible explanation for the differences seen between the transiently transfected cells and the stable, inducible cells, was that these cell lines had become resistant to apoptosis due to the rounds of antibiotic selection required for lineage establishment, as the keratinocyte lines are both G418 (neomycin) and hygromycin resistant. We therefore tested the cell lines with various apoptotic stimuli. Although different cell types vary greatly in their response to various noxious stimuli, ${ }^{21}$ ultraviolet radiation has been shown to reproducibly induce apoptosis in keratinocytes. ${ }^{22,23}$ Therefore, UVB was used initially to test the apoptotic capabilities of the cell lines. The filaggrin expressing lines consistently rounded up and lifted off from the culture dish at lower doses of UVB and at shorter time points compared to the $\beta$-galactosidase expressing lines (Figure 1c,d). After $25 \mathrm{mj}$ UVB, the filaggrin expressing keratinocytes lifted from the culture dish and dissociated from their neighbors (Figure 1c), while the $\beta$ galactosidase expressing keratinocytes showed minimal morphologic effects at the same dose (Figure 1d). At higher doses of UVB or at longer time points, the $\beta$-galactosidase expressing keratinocytes rounded up and separated from the culture dish and their neighbors. Trypan blue staining confirmed that indeed the rounded and floating cells were not viable (data not shown). At $4 \mathrm{~h}$ after exposure to $25 \mathrm{mj}$ UVB, $75 \%$ of the filaggrin expressing keratinocytes were unable to exclude trypan blue (indicating cell death), while only $20 \%$ of the $\beta$-galactosidase expressing keratinocytes were trypan blue positive. With no UVB exposure, both cell populations, either induced with doxycycline or not, exhibited only $0.5-1 \%$ trypan blue positive cells. To differentiate between necrotic and apoptotic death caused by UVB, we employed in situ TUNEL staining and a Cell Death ELISA assay to detect cytoplasmic histone-associated DNA. Results
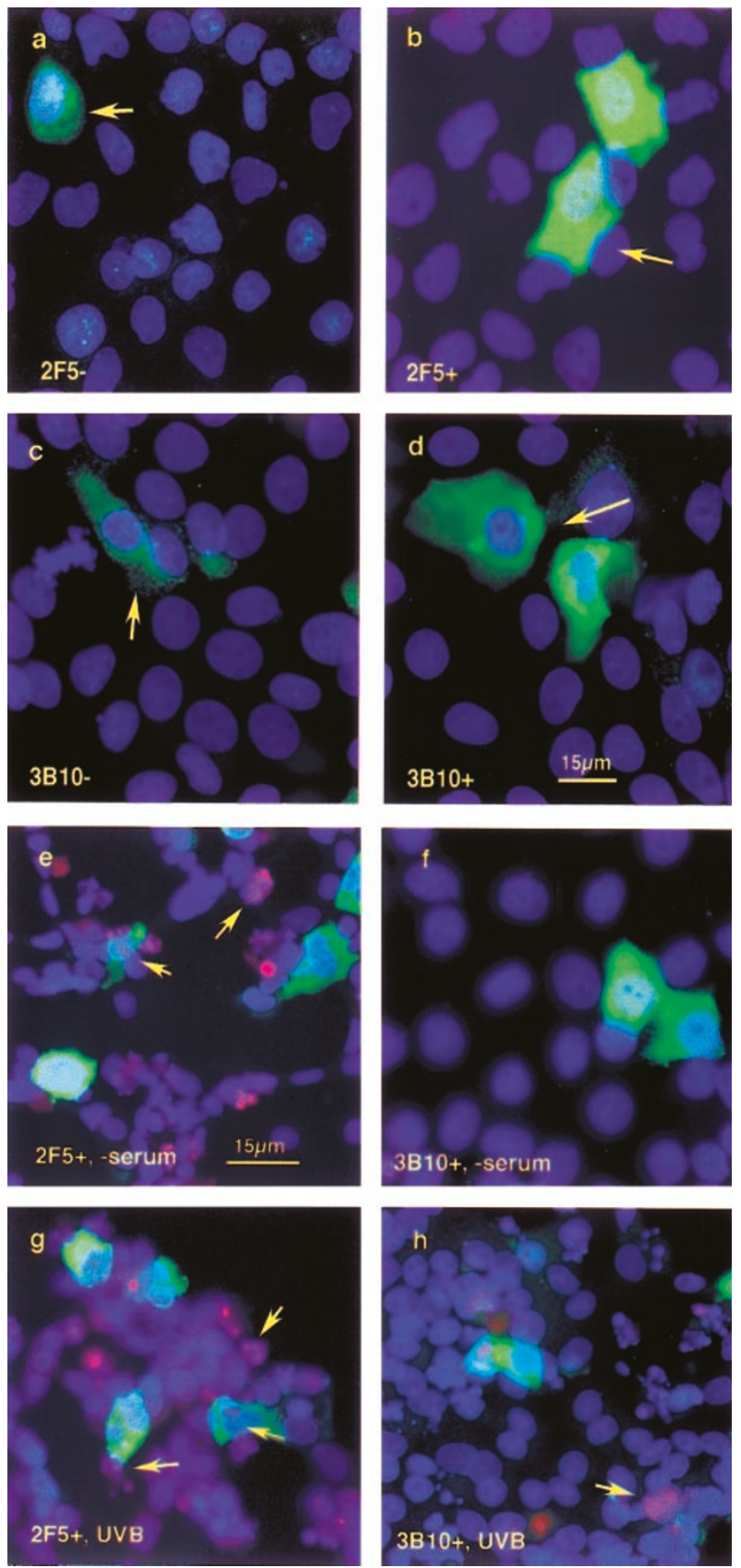

Figure 2 Filaggrin expressing cell lines (2F5) exhibit increased numbers of TUNEL positive nuclei and dysmorphic nuclei when subjected to serum starvation, and UVB exposure compared to $\beta$-gal expressing cell lines (3B10). Cells were grown on coverslips, subjected to the indicated stimulus, fixed in $4 \%$ paraformaldehyde, then subjected to TUNEL staining followed by costaining with either antibody to human filaggrin or to $\beta$-galactosidase. While both cell lines exhibit changes consistent with apoptosis, the filaggrin cell lines (2F5) display the changes to a much greater degree. Panels $\mathbf{a}-\mathbf{d}$ show that there are no TUNEL positive cells in either the filaggrin-expressing line or the $\beta$ galactosidase expressing line with induction of protein alone. Note that there is some expression of the construct in the noninduced cells (a,c, arrows), suggesting some leakiness of the tet-inducible system. Panels $\mathbf{e}$ and $\mathbf{f}$ compare the two induced cell lines after $24 \mathrm{~h}$ of serum starvation. Note that there are numerous TUNEL positive cells in the filaggrin-expressing line (e), but no TUNEL positive cells in the $\beta$-galactosidase expressing line (f). Panels $\mathbf{g}$ and $\mathbf{h}$ compare the results of the two lines $4 \mathrm{~h}$ after exposure to $25 \mathrm{mj}$ UVB. Note that there are numerous TUNEL positive cells in the filaggrin-expressing line $(\mathbf{g})$, compared to the cells in the $\beta$-galactosidase expressing line (h) 
by both methods confirmed that death induced by UVB was from an apoptotic process. Figure 2 shows the results of in situ TUNEL (rhodamine secondary, red) and either filaggrin or $\beta$ galactosidase containing (fluoroscein secondary, green). Inducible expression of either filaggrin or $\beta$-galactosidase alone did not lead to detectable apoptotic nuclei (Figure $2 \mathrm{a}-$ d). When exposed to UVB, however, the filaggrin-expressing cells exhibited many more apoptotic nuclei compared to the $\beta$ galactosidase expressing cells (Figures $2 \mathrm{~g}, \mathrm{~h}, 3 \mathrm{c}, \mathrm{f}$ ).

The response of the filaggrin and control $\beta$-galactosidase REK lines to three additional apoptotic stimuli were examined: serum starvation to represent growth factor withdrawal, cycloheximide to represent a toxin, and hydrogen peroxide, to represent an intermediary in oxidative stress-induced apoptosis. ${ }^{24}$ Apoptosis was assessed in induced and non-induced cells by the Cell Death ELISA assay, by DAPI staining of nuclei, and by the TUNEL assay. The results showed that the filaggrin expressing lines have a greater propensity to undergo apoptosis in response to serum withdrawal and $\mathrm{H}_{2} \mathrm{O}_{2}$ than did control $\beta$-galactosidase lines (Figure 4). After exposure to $\mathrm{H}_{2} \mathrm{O}_{2}$, the filaggrin expressing keratinocytes were completely dissociated from each other and from the culture dish (Figure 1e) and hence, only the Cell Death ELISA that did not require adherent cells was possible (Figure 4)
In some experiments, there was a small (but in most cases not statistically significant) increase in apoptosis in noninduced filaggrin lines compared to either non-induced or $\beta$ galactosidase lines. This may reflect the presence of small numbers of filaggrin positive cells (about $1 \%$ ) present in noninduced cultures that are detected by immunofluorescence (Figure 2a), but not by immunoblot (Figure 6a). Cells expressing filaggrin as determined by immunofluorescence, whether induced or noninduced, demonstrate a higher percentage of apoptotic nuclei than filaggrin negative cells, and $\beta$-galactosidase positive or negative cells (Figure 5). Furthermore, the degree of filaggrin positive cells as determined by immunofluorescence may be underestimated, due to masking of antigenic sites by its keratin association. ${ }^{18,20}$ Nevertheless, filaggrin negative cells clearly undergo apoptosis as a result of serum starvation, UVB exposure and $\mathrm{H}_{2} \mathrm{O}_{2}$, as do the $\beta$-galactosidase cell lines (Figure $2 \mathrm{e}, \mathrm{f}, \mathrm{g}, \mathrm{h}$ ), albeit to a lesser degree. Additional, as yet uncharacterized features of these cell lines may account for a portion of the apoptotic tendency of these cells, but our data clearly show that filaggrin expression is distinctly associated with increased susceptibility to multiple apoptotic stimuli.

Cycloheximide did not lead to apoptosis, regardless of filaggrin expression (Figure 4). This suggests that either the cells are not capable of cycloheximide-induced apoptosis or that filaggrin increases sensitivity to apoptosis in a selective
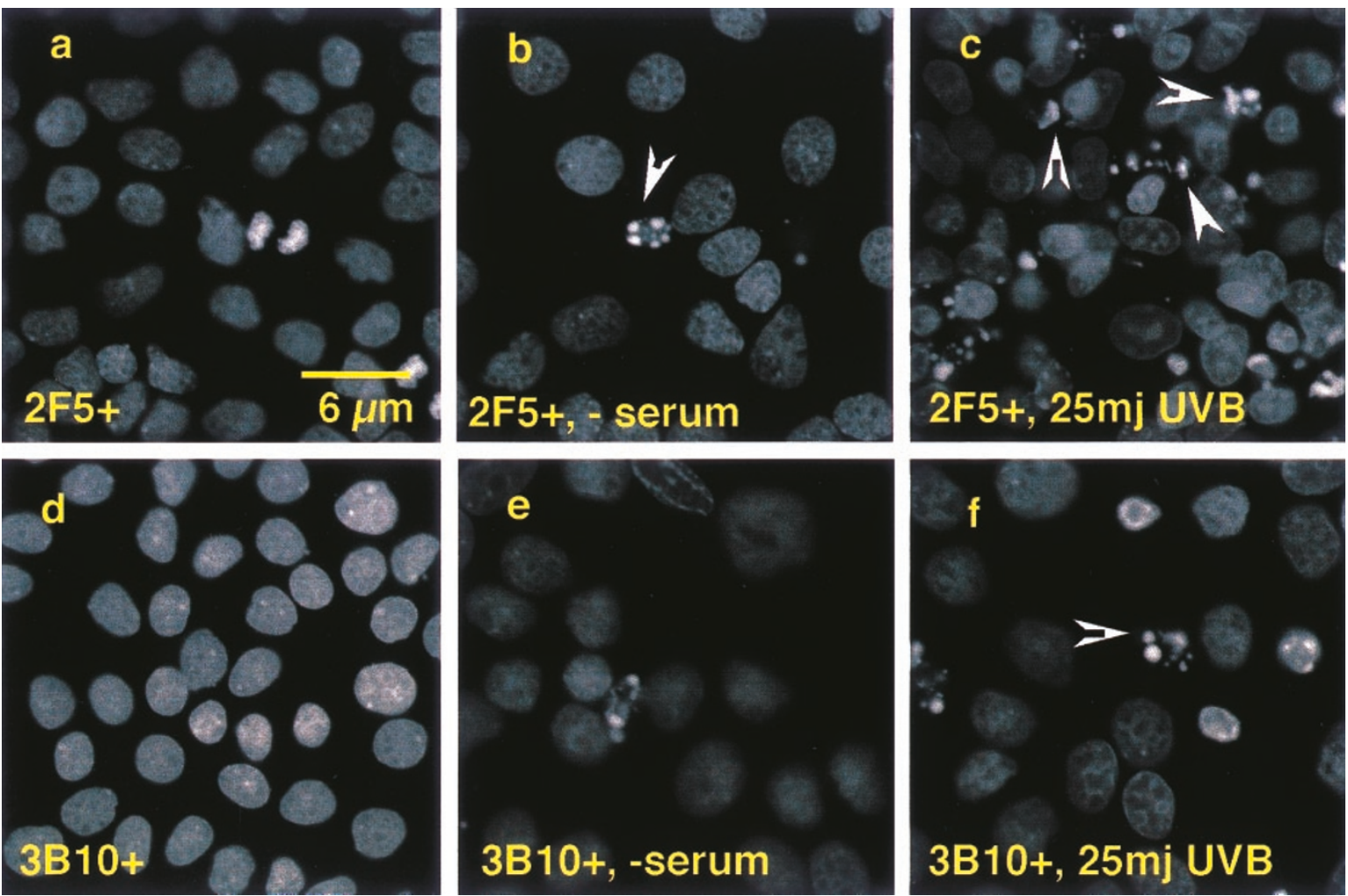

Figure 3 Filaggrin expressing cell lines demonstrate greater numbers of dysmorphic nuclei compared to $\beta$-galactosidase cell lines when subjected to serum starvation and UVB. Cells were grown on coverslips and treated as in Figure 2. DAPI stain is shown in black and white for clarity. Arrows point to apoptotic nuclei, showing condensation or fragmentation. These changes were scored as positive for calculating percentages in Figure 5 . The altered nuclei in panel a are not apoptotic, but are the daughter nuclei of a dividing cell 
manner. The doses and time points of the various apoptotic stimuli presented represent the doses and time points at which apoptosis was readily detected in the filaggrin, but not the $\beta$-galactosidase, cell lines. At higher doses or longer time points, the $\beta$-galactosidase cell lines demonstrated apoptosis whereas the filaggrin cell lines exhibited mainly debris. Both filaggrin and $\beta$-galactosidase lines died by non-apoptotic mechanisms upon exposure to high doses of cycloheximide $(>500 \mu \mathrm{M})$, as measured by Trypan blue exclusion.

\section{Apoptosis is due to inducible filaggrin expression and not endogenous filaggrin formation}

The rat keratinocyte cell line used to establish the stable TETresponsive cell lines is grown in a high calcium media (DMEM

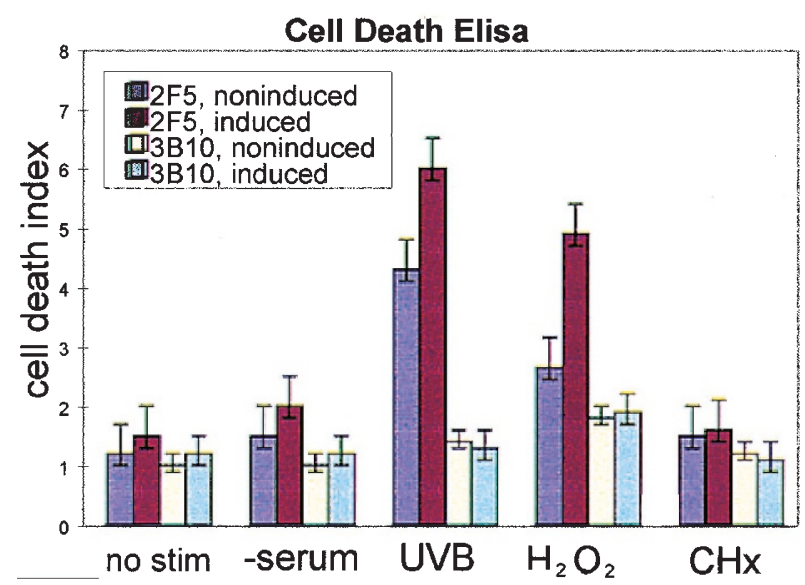

Figure 4 Filaggrin expressing cell lines subjected to serum starvation, UVB and $\mathrm{H}_{2} \mathrm{O}_{2}$ contain a greater amount of cytoplasmic histone-associated DNA compared to $\beta$-gal lines. Cells were grown to $70-90 \%$ confluence and subjected to the indicated apoptotic stimulus. Floating and adherent cells were counted to obtain equivalent numbers of cells per assay condition, then treated as described in Materials and Methods. Experiments were repeated at least three times per condition. Standard error is represented by bars. The higher base-line in non-induced filaggrin line 2F5 may be due to the slight leakiness of the promoter (see Figure 2)

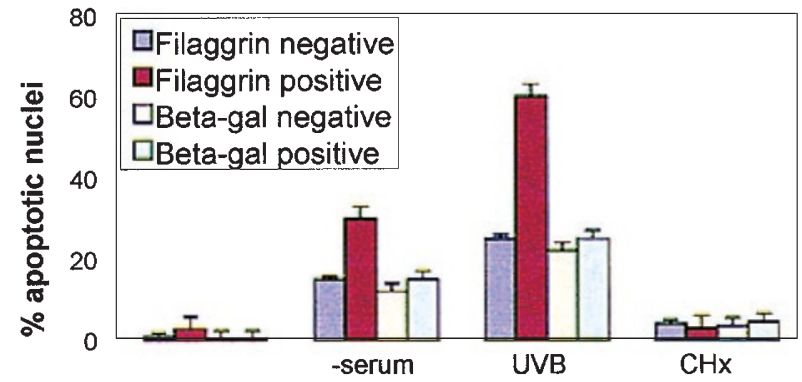

Figure 5 Filaggrin expressing cells exhibit increased numbers of dysmorphic nuclei when subjected to serum starvation, and UVB exposure compared to $\beta$ galactosidase expressing cell lines. To obtain percentages, 10 fields of 200 cells each, both induced and noninduced cells, were counted and scored for the presence of condensed chromatin and fragmented nuclei (DAPI stain). The numbers of filaggrin or -galactosidase positive cells in the noninduced condition remained between 0.5 and $1 \%$ whether subjected to apoptotic stimuli or not. However, the filaggrin positive cells, even in the noninduced cultures, exhibited a higher percentage of apoptotic nuclei with $10 \%$ serum, $2.0 \mathrm{mM} \mathrm{Ca}^{2+}$ ). The cells grow to confluence and begin to stratify, expressing profilaggrin in the stacked cells. Processing of profilaggrin to filaggrin intermediates may occur in post-confluent cultures. UVR, calcium levels, and presence or absence of growth factors have all been shown to affect expression of differentiation markers in human keratinocytes as well as to affect apoptosis. ${ }^{23,25,26}$ To evaluate whether the increased susceptibility to apoptosis in the filaggrin-expressing REK lines was due to the inducible expression of human filaggrin rather than induction of endogenous rat filaggrin, we evaluated the cell lines for expression of rat filaggrin after apoptotic stimuli by immunoblotting. Figure 6 demonstrates that there is no increase in either the tetracycline-inducible human filaggrin or endogenous rat filaggrin after various apoptotic stimuli. A small amount of rat profilaggrin was seen in both the induced and noninduced cell lines that were not subjected to apoptotic stimuli (Figure 6b), but no mature filaggrin was seen with any condition (identical results were obtained the $\beta$-galactosidase expressing lines; data not shown). Interestingly, serum starvation and exposure to $\mathrm{H}_{2} \mathrm{O}_{2}$ led to nonspecific degradation of profilaggrin, but did not result in mature filaggrin. These intermediates probably do not contribute to the apoptosis seen upon induction of these cells, as the intermediates are present in both induced and noninduced cultures. Furthermore, the cultures that were exposed to UVB exhibit no similar intermediates, and UVB was associated with the greatest degree of apoptosis. These data confirm that the increased susceptibility to apoptosis seen with growth factor withdrawal, UVB exposure, and hydrogen peroxide are due to expression of inducible human filaggrin, and not to expression of endogenous rat filaggrin.

\section{a: anti-human fllaggrin (8959)}

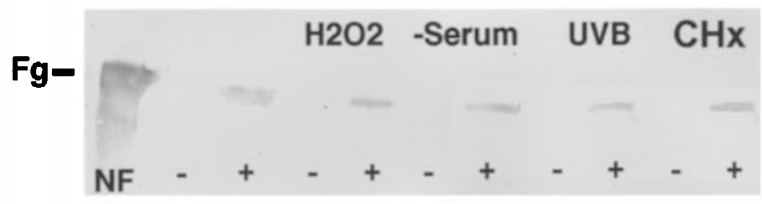

b: antl-rat filaggrin (466)

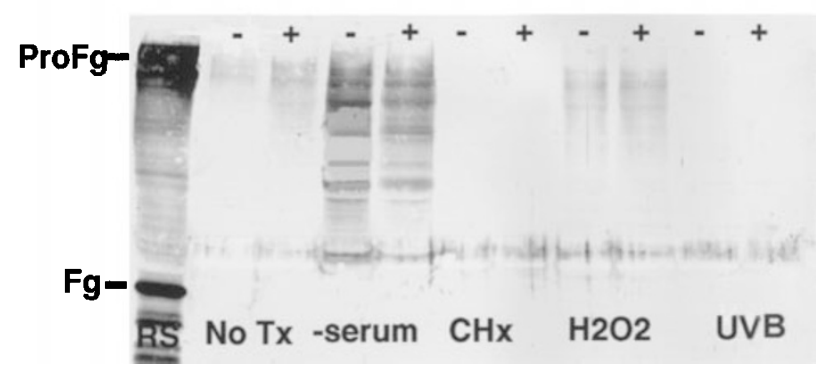

Figure 6 Serum starvation, UVB, $\mathrm{H}_{2} \mathrm{O}_{2}$, and cyclohexamide do not increase expression of either tetracycline-inducible human filaggrin or endogenous rat filaggrin. Urea/tris extracts of cells subjected to the indicated stimulus were separated by $7.5-12 \%$ SDS - PAGE, transferred to nitrocellulose then probed with an antibody to human filaggrin $(8959$, a) or rat filaggrin $(466, \mathbf{b})$. NF, human neonatal foreskin control; RS, rat skin control 


\section{Discussion}

We demonstrate in this study that expression of mature human filaggrin, a protein normally expressed in the epidermis at the time of terminal differentiation (i.e. loss of nucleus and cellular organelles), facilitates induction of apoptosis in keratinocytes. This finding is especially relevant when viewed in the context of the limited expression and specialized function of filaggrin to aggregate the keratin intermediate filament network. Filaggrin is sequestered as an inactive precursor, profilaggrin, prior to its processing to its functional, keratin-binding, form. That filaggrin aids induction of apoptosis in vitro, suggests that it may function in vivo at the granular to stratum corneum transition by enhancing apoptotic machinery.

\section{Apoptosis and terminal differentiation}

Keratinocytes in the granular layer exhibit degraded DNA, ${ }^{13}$ suggesting that endonucleases are active in the transition from granular keratinocyte to corneocyte. Transition cells also lose all other cellular organelles, and cease metabolic function. However, corneocytes are not phagocytized, nor do they exhibit membrane blebbing, both additional morphologic criteria of apoptosis. The lack of these latter two findings is probably due to the presence of the rigid cell envelope, a unique structure not found in other tissues. Nevertheless, it remains unclear if terminal differentiation of epidermal keratinocytes is a form of apoptosis.

The terminal differentiation of lens epithelium parallels in many respects the terminal differentiation of the skin. As lens epithelium differentiates, the precursor cells lose their nuclei and other organelles, and the remaining cellular shell remains in place to perform a function for several weeks after this process. Caspases have been shown to participate in lens epithelial differentiation. ${ }^{27}$ Many caspases are expressed in the epidermis, ${ }^{17}$ and there is evidence for caspase-3 activation in normal human keratinocytes and epidermis. ${ }^{18}$ However, the factors that initiate caspase activation and the site in the epidermis at which they become activated are not yet clear.

Keratinocytes are capable of undergoing apoptosis in vivo in a variety of disease states and in response to UVB. ${ }^{28}$ Often the implication of apoptosis is based on morphology of the dyskeratotic keratinocytes found within the lower spinous layers. Viard et $a l^{29}$ have recently shown involvement of the CD95 (FAS) pathway in toxic epidermal necrolysis (TEN), a full-thickness, desquamative process of the epidermis secondary to a drug reaction. This is the first report linking the apoptotic morphology of the keratinocytes to a known apoptotic pathway. However, in diseases such as TEN, lichen planus, and graft versus host disease where apoptotic keratinocytes are seen, the putative apoptotic keratinocytes are located in the lower layers of the epidermis. One possible explanation for the apparent absence of apoptotic keratinocytes in the granular layer is that the concurrent expression of the cornified cell envelope in the upper layers of the epidermis renders the keratinocyte rigid, therefore preventing the dyskeratosis seen in the lower layers of the epidermis in the context of disease. It is also probable that apoptosis triggered by inflammatory conditions or UV irradiation function through different pathways than those of terminal differentiation.

While expression of filaggrin alone does not lead to apoptosis in the stable cell lines, many filaggrin positive cells exhibit collapsed cytoplasm and altered cytokeratin networks with disrupted desmosomal contacts. ${ }^{19}$ Cell adhesion has been shown to be an important regulator of apoptosis. ${ }^{30}$ We postulate that the filaggrin-expressing cells are more susceptible to apoptotic stimuli due to their compromised cell-cell contacts. ${ }^{19}$ The apparent apoptotic changes seen in the transiently transfected cells can be explained, in part, by the apoptotic potential of the DNAdelivering lipid. ${ }^{31}$

\section{Filaggrin and terminal differentiation}

Filaggrin is derived from a large precursor protein, profilaggrin. Profilaggrin is not able to bind keratins, whereas filaggrin and its smaller peptide derivatives readily bind keratin. 4,20,32 The transition from a granular precursor, profilaggrin, to a diffusely distributed protein happens quickly at the granular to stratum corneum transition in response to an initiating signal which is not yet known. That profilaggrin is expressed as a precursor, rather than a mature protein, suggests that filaggrin expression must be regulated to prevent cytotoxic effects.

Many inflammatory skin conditions are characterized by attenuation of the granular layer with concomitant parakeratosis, i.e. retained nuclei in the keratinocytes of the stratum corneum. ${ }^{33}$ While the signals that are disrupting terminal differentiation in these inflammatory conditions may be disparate, a common final theme is loss of the granular layer with subsequent incomplete terminal differentiation. Filaggrin expression and/or profilaggrin processing is also disrupted in ichthyotic disorders. ${ }^{34-36}$ These disorders are characterized by a markedly thickened stratum corneum. This lends further support to a role for filaggrin in terminal differentiation.

These studies present evidence that expression of filaggrin increases cellular susceptibility to apoptosis in addition to its function in cytoskeletal rearrangement. The biologic significance of this finding lies in the fact that filaggrin is normally expressed at the granular to stratum corneum transition, an area with numerous features of apoptosis. Diseases with perturbed expression of filaggrin exhibit altered terminal differentiation. We hypothesize that filaggrin aids terminal differentiation by potentiating endogenous apoptotic machinery.

\section{Materials and Methods}

\section{Cell culture and generation of stables}

The establishment and characterization of rat epidermal keratinocyte (REK) cell lines that express either filaggrin or $\beta$-galactosidase under tetracycline control ${ }^{37}$ have been reported previously. ${ }^{19}$ The cell lines referred to as 2F5 and 3F5 express human filaggrin, and the cell lines referred to as $2 \mathrm{~B} 5$ and 3B10 express $\beta$-galactosidase.

Selected lines were maintained in 20\% DMEM and passaged weekly. For experimental analyses, REK were seeded at $4 \times 10^{4}$ cells per $60 \mathrm{~mm}$ dish and $1 \times 10^{4}$ cells per well of a 12-well plate, each well 
containing a glass coverslip. Stable REK were grown in 10\% DMEM plus G418 and hygromycin. Two days after plating, doxycycline $(1 \mu \mathrm{g} /$ $\mathrm{ml}$, Sigma Chemical Co., St. Louis, MO, USA) was added to appropriate samples to induce filaggrin expression. Cultures were grown to $75-90 \%$ confluence (either induced or noninduced) before being subjected to apoptotic stimulus. After the apoptotic stimulus, cells were refed the appropriate media until harvesting. Cells on coverslips were washed in PBS, fixed in $4 \%$ paraformaldehyde for $20 \mathrm{~min}$ at room temperature, then stored in PBS at $4^{\circ} \mathrm{C}$ until use. Cells grown in $60 \mathrm{~mm}$ culture dishes were removed via $0.15 \%$ trypsin/EDTA, counted on a hemocytometer, washed in PBS, then stored as a pellet at $-20^{\circ} \mathrm{C}$ until use. Cell viability was evaluated by Trypan blue staining.

\section{Apoptotic stimuli}

Several methods were used to induce apoptosis in the keratinocyte cell lines.

Ultraviolet $B$ radiation An FS20 bulb that emits at a single wavelength of $300 \mathrm{~nm}$ was used to deliver UVB (UltraLum, Paramount, CA, USA). UV intensity was measured with an ultraviolet intensity meter prior to each use (UltraLum, Paramount, CA, USA). Cells were washed in PBS, exposed to UVB for the indicated dose (covered with a thin layer of PBS during exposure to prevent cell drying), then refed appropriate media, and incubated for varying times.

Serum starvation Cells were washed twice with PBS to remove serum, refed DMEM without serum, then incubated for varying times.

Hydrogen peroxide Hydrogen peroxide, $100 \mu \mathrm{M}$ (Sigma Chemical Co., St. Louis, MO, USA), was added directly to the appropriate culture for the indicated time.

Cycloheximide Cycloheximide, $100 \mu \mathrm{M}$ in ethanol (Sigma Chemical Co., St. Louis, MO, USA), was added directly to the appropriate culture for the indicated time. The same volume of ethanol vehicle alone was added to a control culture.

\section{Apoptosis assays}

TUNEL assay For Tdt-mediated dUTP-biotin nick end labeling (TUNEL) analysis, ${ }^{38}$ paraformaldehyde-fixed cells on coverslips were stained using the ApopTag kit (Oncor, Gaithersburg, MD, USA). After the enzymatic labeling step, cells were fixed in $70 \%$ ethanol to facilitate detection of either filaggrin or $\beta$-galactosidase. Visualization of dUTP-biotin labeled nuclei was via a rhodamine-streptavidin secondary antibody.

Nuclear morphologic changes These were evaluated by DAPI (4',6diamidino-2-phenylindole dihydrochloride) staining. Paraformaldehyde-fixed cells were stained with $0.001 \%$ DAPI in TBS for $10 \mathrm{~min}$ at room temperature.

Cell death detection ELISA The cell death ELISA kit was used to detect cytoplasmic histone-associated DNA fragments (Boehringer Mannheim, Indianopolis, IN, USA). The assay was done according to the manufacturer's instructions with the following changes. Both the cells floating in media as well as adherent cells were used. The cells obtained from the different conditions were equilibrated according to cell counts obtained at trypsinization (combined floating and adherent cells). $5 \times 10^{4}$ cells of each condition were lysed in lysis buffer, and
$20 \mu$ l of lysate was used for each reaction. The ELISA plate was read at $405 \mathrm{~nm}$ using a platereader from Molecular Devices Corp equipped with Softmax software (Sunnydale, CA, USA).

\section{Detection of constructs}

Gels and blots Cultured cells transfected with filaggrin constructs were extracted with $0.25 \mathrm{M}$ Tris $/ \mathrm{HCl} \mathrm{pH} 7.8$ in the absence of detergent or urea, and proteins separated on $7.5-12 \%$ gradient SDS-PAGE gels. Tris/urea extracts of human foreskin epidermis or rat epidermis that contain profilaggrin and filaggrin were used as controls. ${ }^{39,40}$ Proteins were blotted to nitrocellulose and immunoreactive proteins visualized as previously described with the avidinbiotin method using 4-chloro-1-naphthol.

Immunofluorescence Polyclonal antibodies (8959) to human filaggrin, and the polyclonal antibody to rat profilaggrin/filaggrin (466) were developed by our laboratory. ${ }^{41,42}$ Anti- $\beta$-galactosidase monoclonal antibody was purchased from Gibco/BRL (Gaithersburg, MD, USA). Paraformaldehyde-fixed cells on coverslips that had previously been subjected to terminal-deoxy transferase were washed in $70 \%$ ethanol for 10 min at $-20^{\circ} \mathrm{C}$, washed three times in PBS, then stained with the appropriate primary antibody for $1 \mathrm{~h}$ at room temperature $(1: 500$ dilution). FITC-labeled goat anti-rabbit (8959) or horse anti-mouse (anti- $\beta$-galactosidase) antibody ( $1: 200$, Vector, Burlingame, CA, USA) were applied for $1 \mathrm{~h}$ at room temperature. Samples were coverslipped using Vectashield mounting medium (Vector, Burlingame, CA, USA) to minimize fading. Image acquisition was on a Nikon Microphot-SA microscope equipped with a CCD camera (Photometrics, Tucson, AZ, USA). Data were collected either as a Z series ( $0.2 \mu \mathrm{M} /$ step) or single 1024 images. The sequentially recorded gray scale images were deconvoluted, pseudocolored, and merged using the software program IPLab (Signal Analytics Corp., Vienna, VI, USA).

\section{References}

1. Marks R (1991) Mechanical properties of the skin. In: Physiology, Biochemistry, and MolecularBiology of the Skin, Goldsmith LA ed (New York: Oxford University Press) pp. 602-621

2. Ishida-Yamamoto $A$ and lizuka $H$ (1998) Structural organization of cornified cell envelopes and alteration in inherited skin disorders. Exp. Dermatol. 7: 1-10

3. Dale BA, Holbrook KA and Steinert PM (1978) Assembly of stratum corneum basic protein and keratin filaments in macrofibrils. Nature 276: 729-731

4. Haugen-Scofield J, Resing KA and Dale BA (1988) Characterization of an epidermal phosphatase specific for filaggrin phosphorylated by casein kinase II. J. Invest. Dermatol. 91: 533-559

5. Kam E, Resing KA, Lim S and Dale BA (1993) Identification of rat epidermal profilaggrin phosphatase as a member of the protein phosphatase $2 \mathrm{~A}$ family. J. Cell. Sci. 106: 219-226

6. Resing KA, Walsh KA and Dale BA (1984) Identification of two intermediates during processing of profilaggrin to filaggrin in neonatal mouse epidermis. J. Cell. Biol. 99: 1372-1378

7. Resing KA, Walsh KA, Haugen-Scofield J and Dale BA (1989) Identification of proteolytic cleavage sites in the conversion of profilaggrin to filaggrin in mammalian epidermis. J. Biol. Chem. 264: 1837-1845

8. Haake AR and Polakowska RR (1993) Cell death by apoptosis in epidermal biology. J. Invest. Dermatol. 101: 107-112

9. Maruoka Y, Harada H, Mitsuyasu T, Seta Y, Kurokawa H, Kajiyama M and Toyoshima K (1997) Keratinocytes become terminally differentiated in a process involving programmed cell death. Biochem. Biophys. Res. Commun. 238: 886890 
10. Tamada Y, Takama H, Kitamura T, Yokochi K, Nitta Y, lkeya T and Matsumoto $Y$ (1994) Identification of programmed cell death in normal human skin tissues by using specific labeling of fragmented DNA. Br. J. Dermatol 131: 521-524

11. Ishida-Yamamoto A, Takahashi H, Presland RB, Dale BA and lizuka H (1998) Translocation of profilaggrin $\mathrm{N}$-terminal domain into keratinocyte nuclei with fragmented DNA in normal human skin and loricrin keratoderma. Lab. Invest. 78: $1245-1253$

12. McCall CA and Cohen JJ (1991) Programmed cell death in terminally differentiating keratinocytes: role of endogenous endonuclease. J. Invest. Dermatol. 97: 111-114

13. Gandarillas A, Goldsmith LA, Gschmeissner S, Leigh IM and Watt FM (1999) Evidence that apoptosis and terminal differentiation of epidermal keratinocytes are distinct processes. Dermatol. 8: 71-79

14. Cohen G (1997) Caspases: the executioners of apoptosis. Biochem. J. 323: 1 16

15. Villa P, Kaufman SH and Earnshaw WC (1997) Caspases and caspase inhibitors. TIBS. 22: 388-393

16. Takahashi T, Ogo $M$ and Toshihiko $H$ (1998) Partial purification and characterization of two distinct types of caspases from human epidermis. J. Invest. Dermatol. 111: $367-372$

17. Weil M, Raff MC and Braga VMM (1999) Caspase activation in the terminal differentiation of human epidermal keratinocytes. Curr. Biol. 9: 361-364

18. Dale BA, Presland RB, Lewis SP, Underwood RA and Fleckman P (1997) Transient expression of epidermal filaggrin in cultured cells causes collapse of intermediate filament networks with alterations of cell shape and nuclear integrity. J. Invest. Dermatol. 108: 179-187

19. Presland RB, Kuechle MK, Lewis SP, Fleckman P and Dale BA. Regulated expression of filaggrin in keratinocytes results in cytoskeletal disruption, loss of cell-cell adhesion, and cell cycle arrest, but not apoptosis. Submitted with revisions to J Cell Science

.20 Kuechle MK, Thulin CD, Presland RB and Dale BA (1999) Profilaggrin requires both linker and filaggrin peptide sequences to form granules: Implications for profilaggrin processing in vivo. J. Invest. Dermatol. 112: 843-852

21. McGahon AJ, Martin SJ, Bissonnette RP, Mahboubi A, Shi Y, Mogil RJ, Nishioka WK and Green DR (1995) The end of the (cell) line: methods for the study of apoptosis in vitro. Methods in Cell Biology 46: 153-185

22. Benassi L, Ottani D, Fantini F, Marconi A, Chiodino C, Giannetti A and Pincelli C (1997) 1,25-dihydroxyvitamin $\mathrm{D}_{3}$, transforming growth factor $\beta 1$, and ultraviolet $\mathrm{B}$ radiation induces apoptosis in cultured human keratinocytes. J. Invest. Dermatol. 109: 276-282

23. Norris DA, Middleton MH, Whang K, Schleicher M, McGovern T, Bennion SD, David-Bajar K, Davis D and Duke RC (1997) Human keratinocytes maintain reversible anti-apoptotic defenses in vivo and in vitro. Apoptosis 2: 136-148

24. Goel R and Khanduja KL (1998) Oxidative stress-induced apoptosis-An overview. Current Science 75: $1338-1345$

25. Bernerd F and Asselineau D (1997) Successive alteration and recovery of epidermal differentiation and morphogenesis after specific UVB-damages in skin reconstructed in vitro. Dev. Biol. 183: 123-138

26. Kumar MG, Hurwitz SA, Cotton J and Spandau DF (1999) Subphysiologic concentrations of extracellular calcium sensitize normal human keratinocytes to UVB-induced apoptosis. Arch. Dermatol. Res. 291: 37-46
27. Ishizaki Y, Jacobson MD and Raff MC (1998) Role for caspases in lens fiber differentiation. J. Cell. Biol. 140: 153-158

28. Haake AR and Polakowska RR (1997) Pathways to programmed cell death: apoptosis and the skin. Curr. Opin. in Dermatol. 4: 247-255

29. Viard I, Wehrli P, Bullani R, Schneider P, HollerN, Salomon D, Hunziker T, Saurat $\mathrm{JH}$, Tschopp J and French LE (1998) Inhibition of toxic epidermal necrolysis by blockade of CD95 with human intravenous immunoglobulin. Science 282: 490 493

30. Ruoslahti E and Reed J (1994) Anchorage dependence, integrins, and apoptosis. Cell 77: $477-478$

31. LiXL, Boyanapalli M, Xiao WH, Kalvakolanu DV and Hassel BA (1998) Induction of interferon synthesis and activation of interferon-stimulated genes by liposomal transfection reagents. J. Interferon and Cytokine Res. 18: 947-952

32. Mack JW, Steven AC and Steinert PM (1993) The mechanism of interaction of filaggrin with intermediate filaments: the ionic zipper hypothesis. J. Mol. Biol. 232 $50-66$

33. Lever WE and Schaumberg-Lever G. Histopathology of the Skin, 7th ed Lippincott, Philadelphia, PA. 1990

34. Dale BA, Resing KA and Haydock PV (1990) Filaggrins. In Cellular and Molecular Biology of Intermediate Filaments, Goldman RD and Steinert PM, eds (New York: Plenum Publishing Corp) pp. 393-412

35. Manabe M, Sanchez M, Sun T-T and Dale BA (1991) Interaction of filaggrin with keratin filaments during advanced stages of normal human epidermal differentiation and in ichthyosis vulgaris. Differentiation 48: 43-50

36. Fleckman P and Dale BA (1993) Structural protein expression in the ichthyoses. In From Molecular Biology to Therapeutics. Pharmacol Skin. Bernard BA, Shroot B eds (Basel: Karger) pp. 1-15

37. Gossen MS, Freundlieb G, Bender G, Muller G, Hillen W and Bujard H (1995) Transcriptional activation by tetracyclines in mammalian cells. Science 268 : 1766- 1769

38. Gavrieli Y, Sherman Y and Ben S-SA (1992) Identification of programmed cell death in situ via specific labeling of nuclear DNA fragmentation. J. Cell. Biol. 119: 493-501

39. Laemmli UK (1970) Cleavage of structural proteins during the assembly of the head of bacteriophage T4. Nature 227: 680-685

40. Haydock PV, Blomquist C, Brumbaugh S, Dale BA, HolbrookKA and Fleckman P (1993) Antisense profilaggrin RNA delays and decreases profilaggrin expression and alters in vitro differentiation of rat epidermal keratinocytes. J. Invest. Dermatol. 101: 118-126

41. Fleckman P, Dale BA and Holbrook KA (1985) Profilaggrin, a high-molecularweight precursor of filaggrin in human epidermis and cultured keratinocytes. $J$. Invest. Dermatol. 85: 507-512

42. Haydock PV and Dale BA (1986) The repetitive structure of the profilaggrin gene as demonstrated using epidermal profilaggrin cDNA. J. Biol. Chem. 261:12520 12525 